\title{
Early radiographic features in patients with anterior cruciate ligament rupture
}

\author{
J C Buckland-Wright, J A Lynch, B Dave
}

\begin{abstract}
Objective-To determine, in a preliminary cross sectional study of patients with anterior cruciate ligament (ACL) ruptured knees, which of the radiographic features-subchondral cortical plate thickness, trabecular sclerosis, and osteophytosis-appears before or in association with changes in joint space width (JSW) as a surrogate for articular cartilage thickness in patients with ruptured knees.

Methods-19 patients (14 men), mean (95\% CI) age 28.7 (23.6 to 33.8 ) years, had ACL rupture in one knee only, confirmed by arthroscopy. Anterior draw, Lachman's test, and pivot shift tests were performed and $\times 5$ posteroanterior macroradiographs of both knees in a standing semiflexed view were taken at a mean $(95 \% \mathrm{CI})$ time from injury of 34.3 (26.9 to 41.7 ) months. In each radiograph, computerised methods were used to measure minimum JSW and fractal signature analysis (FSA) to quantify the vertical and horizontal trabecular organisation, osteophyte area, and the subchondral cortical plate thickness, in the medial and lateral tibial compartments of all knees.
\end{abstract}

Results-Physical examination confirmed the presence of joint laxity in the injured

Department of Applied Clinical Anatomy, Guy's, King's, and St Thomas's School of Biomedical Science, King's College London, Guy's Campus, London SE1 1UL, UK J C Buckland-Wright J A Lynch

Department of Orthopaedics, Guy's and St Thomas's Hospital Trust, London Bridge, London SE1 9RT, UK B Dave

Correspondence to: Professor J C

Buckland-Wright, Department of Applied Clinical Anatomy, GKT School of Biomedical Science, King's College, Hodgkin Building, London Bridge, London SE1 1UL, UK.

E-mail:

chris.buckland-wright@ kcl.ac.uk

Accepted for publication 14 February 2000 knees. No statistical difference in either medial or lateral JSW and subchondral cortical plate thickness was detected between ACL ruptured and uninjured knees. FSA showed a significant decrease in fractal dimension for the horizontal trabeculae (size $0.42 \mathrm{~mm}$ ) in the medial compartment of the ACL ruptured knees compared with the uninjured knees $(p<0.01$, multivariate analysis of variance). There was an inverse correlation ( $R$ $-0.74)$ between the time since knee injury and the difference in FSA values for ACL ruptured and uninjured knees in medial compartment horizontal trabeculae (size $0.4 \mathrm{~mm})$. No significant changes were detected in the lateral compartment. Osteophytes were detected in the medial compartment of nine ACL injured knees. Conclusion-Compared with the uninjured knee, ACL rupture leads to thickening of subchondral horizontal trabeculae (decreased FSA) in the medial tibial compartment of all knees, reaching significance at about four years after injury. Osteophytes were present in the same compartment in 9/19 knees. No changes in JSW and subchondral cortical plate thick- ness were detected. Whether the bony changes detected in these ACL ruptured knees presage those of early osteoarthritis requires further study.

(Ann Rheum Dis 2000;59:641-646)

Retrospective studies in post-traumatic patients with anterior cruciate ligament (ACL) rupture show radiographic changes consistent with osteoarthritis (OA) in $60-90 \%$ of such patients 10-15 years after injury. ${ }^{1-6}$ These patients with a tear of the cruciate ligament, isolated or combined with injuries to the collateral ligament or the menisci, show radiographic signs of $\mathrm{OA}$ at a younger age than patients with a meniscal tear only, ${ }^{7}$ and a faster disease progression than those with primary OA. ${ }^{4}$ The pathogenesis of cartilage damage and OA after joint injury is uncertain. Factors that have been suggested as having a role include chronic instability, resulting in mechanical overload, chronic low grade synovitis, and injury to the joint cartilage and subchondral bone plate in connection with the trauma. ${ }^{8}$ Investigations with magnetic resonance imaging (MRI) have shown that there is an $85 \%$ chance that the ACL ruptures result in reticular subcortical fractures or "bone bruising", referring to oedema and haemorrhage in the medullary bone. ${ }^{9}$ The bone bruises appear principally in the region of the posterolateral tibial plateau and the anterolateral tibial rim, the latter being associated with osseous injuries sustained at the terminal sulcus on the lateral femoral condyle. ${ }^{10}$ These changes, it is suggested, are in conformity with the findings of greatly increased concentrations of markers of cartilage matrix metabolism in joint fluid consequent upon the trauma. ${ }^{11}$

The association between the initial site of bony trauma in the lateral compartment of patients with ACL rupture ${ }^{910}$ and the later appearance of radiographic features of $\mathrm{OA}^{1-6}$ was evaluated using high definition macroradiography. ${ }^{12}{ }^{13}$ The advantages of magnification and high spatial resolution of this method permit the early detection of $\mathrm{OA}$ features. ${ }^{14-17}$ These studies have shown that bony features appear before cartilage narrowing and loss measured radiographically as joint space narrowing. ${ }^{15} 1718$

As post-traumatic OA has the advantage that the beginning of the disease process can be identified by the date of the injury, ${ }^{7}$ quantitative macroradiography was used in this preliminary, cross sectional study to determine the sequence of appearance of the radiographic features relative to one another. In particular, which of the bony features-subchondral 
cortical plate thickness, subchondral trabecular sclerosis, and osteophytosis-occurred before or in association with changes in joint space width as a surrogate for articular cartilage thickness.

\section{Patients and methods}

After we had received Lewisham and North Southwark ethical committee approval, 19 patients (14 men), with mean age (95\% confidence interval (CI)) 28.7 (23.6 to 33.8) years, and a mean $(95 \% \mathrm{CI})$ time since injury of 34.3 (26.9 to 41.7) months, were recruited from those attending the outpatient clinic. The rupture of the ACL was the result of a sports injury in all but two, who sustained their injury in the home. Patients who had ACL rupture only in one knee, confirmed by arthroscopy, and who had elected for conservative treatment were selected. Patients were excluded if they had sustained injury to the meniscus or articular cartilage, or both, at the time of the index trauma. All patients attended the department of physiotherapy as outpatients on average 4.5 (range 1-12) months for treatment and eight patients used a knee brace or elasticated support during periods of increased activity. At the time of the radiographic examination, joint laxity was determined by the anterior draw test, obtained in the neutral position and with internal and external rotation respectively, by Lachman's test, and by pivot shift tests.

PREPARATION OF MACRORADIOGRAPHS

High definition posteroanterior macroradiographs ${ }^{12}{ }^{13}$ of both knees were taken at $\times 5$ magnification in a standing, semiflexed view. ${ }^{19}$ Radiographic magnification for computing the size of $x$ ray features within the joint was determined by placing metal balls (diameter $2 \mathrm{~mm}$ ) on the knee's anterior and posterior surfaces before radiography, and from measurements taken of the focus to knee and focus to film distance after radiography.

\section{Standing semiflexed view}

In the standing semiflexed view ${ }^{19}$ the surface of the tibial plateau is horizontal with the joint positioned close to the normal anatomical standing position and to the region of major contact stresses in the tibiofemoral articulation..$^{20}$ The centre of the joint, defined by the joint space, was aligned with the centre of the $x$ ray beam with the aid of a cross-optic laser. Fluoroscopy was used to study each knee, which was flexed until the tibial plateau was horizontal relative to the floor, parallel to the central $x$ ray beam, and perpendicular to the $x$ ray film, and the tibial spines were centrally placed relative to the femoral notch.

\section{IMAGE ANALYSIS}

The macroradiographs were digitised using a Lumisys $200 \mathrm{HR}$ laser scanner at a pixel size of $0.06 \mathrm{~mm}$ (after correcting for magnification), and the images were stored and analysed with a Sun Sparcstation, model 20/61 (Sun Microsystems Ltd) and programs written in C were used to measure $\mathrm{JSW}^{1521}$ and to calculate the fractal signature $^{22} 23$ for regions of interest in the images.

\section{Foint space width measurement}

For each knee image the minimum joint space width (JSW) in both the medial and lateral compartments was measured with an automated image analysis technique. ${ }^{15}{ }^{21}$ The position and size of the minimum JSW of each compartment was displayed on the computer screen and stored on disk for later analysis. The accuracy and reproducibility of the radiographic procedure and automatic method of JSW measurement and reproducibility of patient repositioning in the standing semiflexed view gave a coefficient of variation for JSW measurement in the medial compartment of $1.2 \%$ and in the lateral compartment of $3.8 \% .^{21}$ All assessments were carried out by one observer whose coefficient of variation for repeat measures for the medial compartment was $0.6 \%$ and for the lateral compartment $3.6 \% .^{21}$

Measurement of subarticular cancellous bone Fractal analysis is a robust method ${ }^{24}$ which is independent of a range of factors that are susceptible to variation during routine radiographic procedures - that is, the effect of radiographic magnification and projection geometry, ${ }^{22} 2325$ changes in object or patient position, ${ }^{22-28}$ and variations in the sensitometric properties of radiographs such as film contrast and mean density. ${ }^{22} 2325$ Figure 1 shows the region of interest (size after correction for radiographic magnification $18 \times 6 \mathrm{~mm}$ ) selected within the digitised macroradiographs of the medial and lateral compartments of the tibia. Within this region of interest fractal signature analysis (FSA) was obtained separately for horizontal and vertical trabecular structures. ${ }^{17}$ As tibial trabecular thickness and medullary space size is in general within 0.06 $\mathrm{mm}$ and $1.2 \mathrm{~mm},{ }^{29}$ the fractal signature calculations were carried out on data ranging from 1 to 20 pixels, corresponding to a scale of image feature sizes from $0.06 \mathrm{~mm}$ to $1.2 \mathrm{~mm}$. We assessed accuracy by determining the variations in measurements of FSA for horizontal and vertical structures from the macroradiograph of a tibia redigitised six times, and for test-retest reliability by digitising the macroradiographs of a postmortem tibia repositioned and reradiographed six times. The coefficient of variation for test-retest for FSA measurements was $2.1 \% .^{23}$

\section{Measurement of osteophytes and subchondral} cortical plate thickness

Measurements were taken using a PC driven Kontron digitisation program (KS-100; Kontron Electronik $\mathrm{GmbH}$, Eching, Germany) linked to a back-illuminated digitisation tablet. The measurements were carried out using a crosswire cursor. The thickness (in millimetres) of the subchondral cortex of the tibia was measured three times at the midpoint of each plateau and the readings averaged for the medial and lateral compartments respectively. The coefficient of variation for this method for 


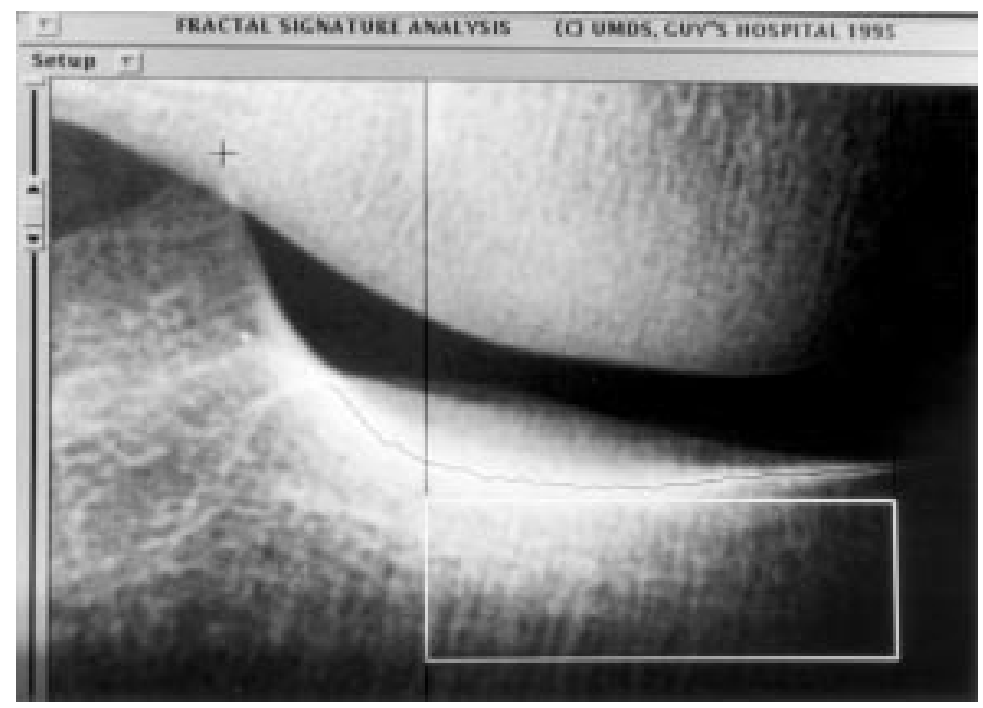

Figure 1 Part of a computer screen showing a digitised macroradiograph of a knee. The region of interest used for calculating the fractal signature of the tibial cancellous bone in the medial compartment is outlined in white; the horizontal black line defines the floor of the tibial plateau.

repeat linear measurements was $9.4 \% .{ }^{15}$ The number of osteophytes at the margins and at the tibial spines in the medial and lateral tibiofemoral compartments was counted and their area in square millimetres measured with the crosswire cursor. The coefficient of variation for repeat osteophyte area measurements was $6.5 \% .^{15}$

DATA ANALYSIS

The mean and $95 \%$ confidence intervals were calculated for the minimum JSW, subchondral cortical thickness, and osteophyte area measurements in the medial and lateral compartments in the ACL ruptured and uninjured knees. The significance of any difference in these measurements in each compartment between the injured and uninjured knees was determined using Wilcoxon signed rank test. The difference in the fractal signature for horizontal and vertical structures respectively, between patients' injured and uninjured knees, in each tibiofemoral compartment was assessed for significance using multivariate analysis of variance with repeat measures. To examine the difference between the FSA of the ACL ruptured and contralateral uninjured knee for each patient, Wilcoxon signed rank test was used. For correlations between time since injury for each patient and each of the measured features Pearson's correlation coefficient (R) was used. Mann-Whitney U test was used to determine the probability that duration

Table 1 Mean and 95\% confidence intervals (CI) for joint space width ( $(F S W)$ and subchondral cortical thickness (SCT), measured in millimetres, in anterior cruciate ligament (ACL) ruptured and uninjured knees in the medial and lateral compartments

\begin{tabular}{|c|c|c|c|c|}
\hline & \multicolumn{2}{|c|}{ Medial compartment } & \multicolumn{2}{|c|}{ Lateral compartment } \\
\hline & $\begin{array}{l}\text { Mean } \\
(\mathrm{mm})\end{array}$ & $\begin{array}{l}95 \% C I \\
(\mathrm{~mm})\end{array}$ & $\begin{array}{l}\text { Mean } \\
(\mathrm{mm})\end{array}$ & $\begin{array}{l}95 \% \text { CI } \\
(\mathrm{mm})\end{array}$ \\
\hline JSW: ACL ruptured knee & 4.83 & 4.30 to 5.36 & 6.39 & 5.85 to 6.93 \\
\hline JSW: uninjured knee & 5.05 & 4.62 to 5.49 & 6.16 & 5.56 to 6.76 \\
\hline SCT: ACL ruptured knee & 0.96 & 0.83 to 1.76 & 0.63 & 0.54 to 0.72 \\
\hline SCT: uninjured knee & 0.96 & 0.81 to 1.22 & 0.62 & 0.55 to 0.70 \\
\hline
\end{tabular}

since injury was related to osteophyte formation in the ACL knee and whether there was an association between osteophyte size and any changes in FSA of trabecular structures detected in the ACL ruptured knees. The method of Cummings and Black ${ }^{30}$ was used to calculate the minimum interval change that could be measured with $95 \%$ confidence. The significance level for all statistical tests was set at $\mathrm{p}=0.05$.

\section{Results}

PHYSICAL EXAMINATION

In the ACL ruptured knee the anterior draw test was positive in all patients with the joint in the neutral position, six patients had a marked anterior displacement. With the knee in external rotation the anterior draw test was positive in $15 / 19$ patients, of whom four had a marked anterior displacement; with the joint in internal rotation the anterior draw test was positive in 11/19 patients, of whom three had a marked anterior displacement. Lachman's test was positive in 17/19 patients, of whom seven had a marked displacement. The pivot shift test was positive in $9 / 19$ patients, unobtainable in three, and absent in the remaining patients. Joint laxity was confirmed by all three tests in seven patients and by two of the tests in the remaining 12 patients.

JOINT SPACE WIDTH

The JSW in the ACL ruptured knees was not statistically significantly different from the contralateral (uninjured) knees in either the medial or lateral compartments (table 1).

\section{SUBCHONDRAL CORTICAL PLATE THICKNESS}

There was no difference in the cortical plate thickness in the medial and lateral compartments of the ACL ruptured compared with the uninjured knee (table 1).

FSA OF HORIZONTAL TRABECULAR STRUCTURES In the medial compartment the fractal signature values for horizontal trabecular structures were significantly lower in the ACL ruptured knees than in the uninjured knees $(p<0.01$, multivariate analysis of variance) (fig 2). This result was confirmed by examining the difference between the FSA of the ACL ruptured and the contralateral (uninjured) knee for each patient. Compared with the contralateral knee, the ACL ruptured knee showed significantly lower FSA values $(p<0.01)$ for horizontal trabeculae at $\sim 0.4 \mathrm{~mm}$ in size (fig 3 ). In the lateral compartment the FSA results showed that there was no significant difference between the ACL ruptured and uninjured knees.

FSA OF VERTICAL TRABECULAR STRUCTURES In both the medial and lateral compartments no statistically significant difference in the FSA values was detected, nor were any differences detected between the ACL ruptured and the uninjured knees.

\section{TIME SINCE INJURY}

The magnitude of the difference in FSA for horizontal trabeculae (at the dimension of 0.4 


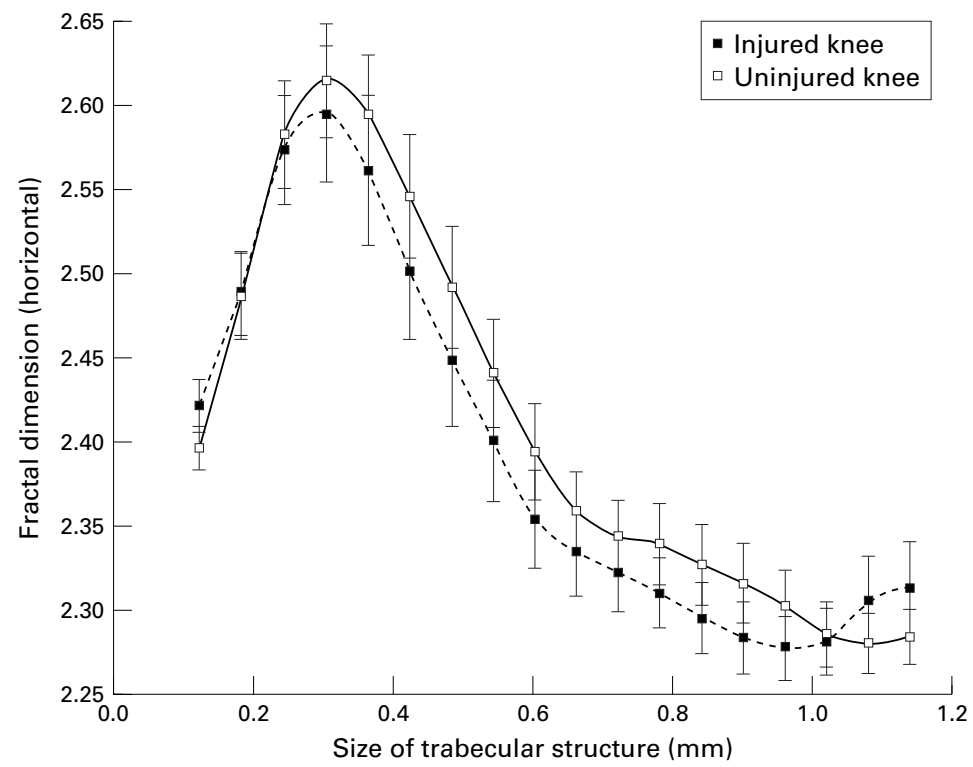

Figure 2 Mean (SEM) of the medial compartment fractal signature analysis of horizontal trabecular structures in the ACL injured and uninjured knees; the difference was significant ( $p<0.01$, multivariate analysis of variance with repeat measures).

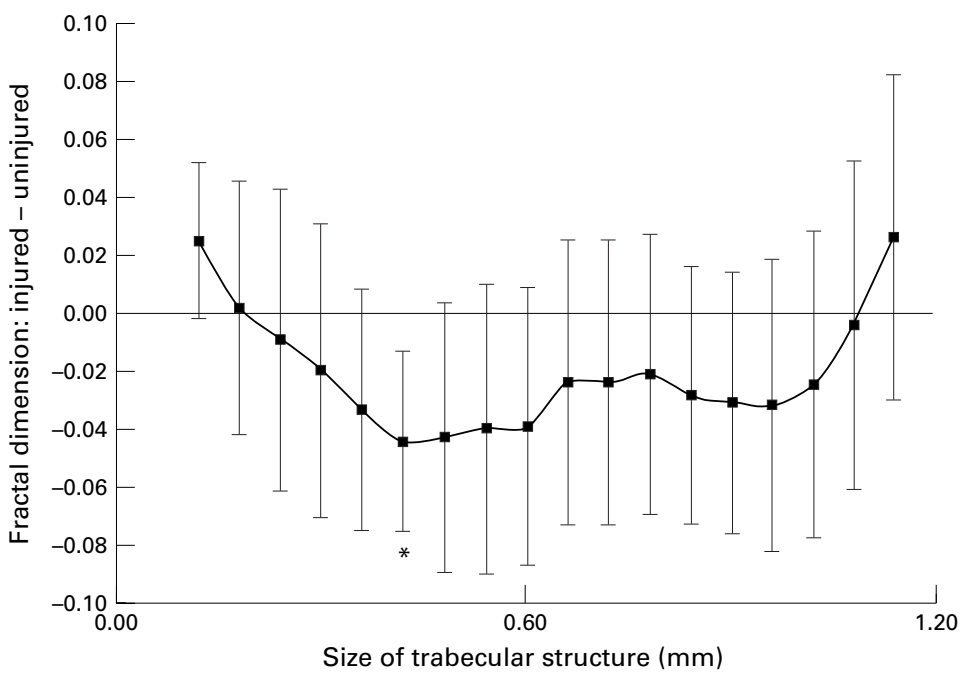

Figure 3 Mean $(95 \%$ CI) difference in medial compartment fractal signature analysis of horizontal trabecular structure between ACL ruptured and contralateral (uninjured) knee. *Indicates the trabecular structures of $0.4 \mathrm{~mm}$ in dimension that were significantly thicker in ACL ruptured knees $(p<0.05)$ than in the uninjured knees.

$\mathrm{mm}$ ) in the medial compartment between ACL ruptured and uninjured knees was significantly correlated with the time since injury $(\mathrm{R}=-0.74, \mathrm{p}<0.01)$ (fig 4$)$. In five patients for whom time since injury was greater than 40 months, the decrease in the FSA for horizontal trabeculae exceeded the reproducibility of FSA measurements $( \pm 0.05) .{ }^{17}{ }^{31}$ The shortest time from injury in which a statistically significant change in FSA was detected was four years (three $(16 \%)$ cases). Neither the joint space width nor the subchondral cortical thickness in the medial and lateral compartments in the damaged knee was related to the time since injury.

OSTEOPHYTOSIS

Osteophytes were observed at the tibial margin and spines in the medial compartment only of

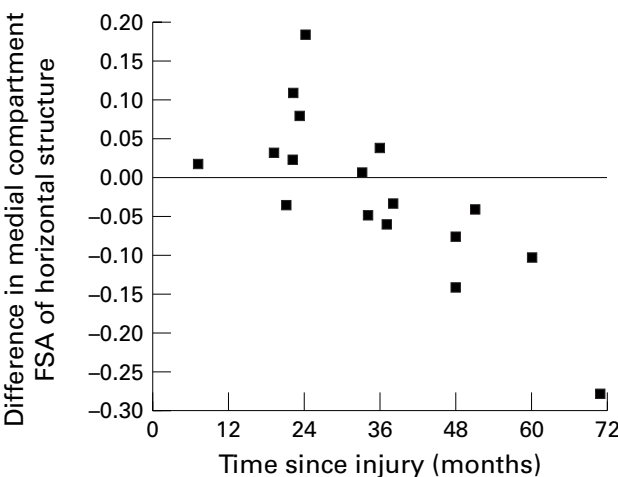

Figure 4 Difference in medial compartment fractal signature analysis (FSA) value for horizontal trabecular structures at $0.4 \mathrm{~mm}$ size between $A C L$ ruptured and contralateral (uninjured) knee in each patient plotted against time since injury. The magnitude of the difference in FSA correlated significantly with time since injury $(R=-0.74, p<0.01)$.

the ACL ruptured knee. Nine of the 19 patients had osteophytes, two patients had a single osteophyte, four patients had two, two patients had three, and one patient had four osteophytes. The mean (95\% CI) osteophyte size was 84.7 (133.6 to 35.8$) \mathrm{mm}^{2}$. Although not statistically significant, osteophyte number, but not area, tended to be greater in those patients who had had the injury longest. The mean ( $95 \%$ CI) for the time since injury in the patients with osteophytes in the medial compartment of their damaged knee was 39 (16.1 to 61.7$)$ months compared with a time of 26 (16.0 to 35.8) months for the patients without osteophytes. No correlation was found between changes in the FSA for horizontal structures and either osteophyte number or size.

\section{Discussion}

High definition macroradiography of patients with post-traumatic injury to the knee of fairly short duration detected anatomical changes to the medial compartment of the injured joint consistent with the early stages of OA reported previously. ${ }^{15} 1718 \mathrm{JSW}$ measurement, as a surrogate measure for articular cartilage thickness, ${ }^{14}$ showed no change compared with the contralateral (uninjured) knee, confirming that changes in cartilage thickness are characteristic of a later stage of the disease, associated with cartilage softening and its subsequent physical loss. ${ }^{15}{ }^{32}$ Nevertheless, changes in the cartilage would have been present because synovial fluid analyses obtained from ACL ruptured knees have shown that the injury results in a disruption of the normal biochemical processes in articular cartilage and that these precede changes in its physical appearance. ${ }^{83}$

Within the subarticular region of the medial compartment of the tibia, the subchondral cortical plate thickness was found to be similar in both the injured and uninjured knees, whereas FSA of the tibial cancellous bone showed that the trabecular structure had altered in the medial compartment only in ACL ruptured knees. The decrease in the fractal signature for the horizontal structures is consistent with an increase in trabecular thickness. ${ }^{171}$ The change in fractal signature reached statistical 
significance for structures at $0.4 \mathrm{~mm}$ in size about four years after injury in three $(16 \%)$ cases. The increased thickness in horizontal trabeculae of this size was the same as that detected in patients with early stage medial compartment knee $\mathrm{OA}^{17}{ }^{31}$ using the same methods. No significant changes in cancellous bone were detected in the lateral compartment of the tibia in the injured knee. This again was consistent with that recorded in patients with OA with medial compartment disease. ${ }^{17} 31$

The results described here differ from those of our previous study of patients with early knee OA. ${ }^{15}{ }^{17}$ In OA knees ${ }^{15}$ the medial compartment tibial subchondral cortical plate was thicker than in the reference non-arthritic group. This would suggest that there is coupling between cortical plate remodelling and early changes in cartilage thickness - an observation that is supported by results from both experimental animal work $^{34}$ and in human hand and knee OA. ${ }^{15} 18{ }^{35}$ In this study no differences in subchondral cortical plate thickness and JSW were found between the injured and uninjured knees, suggesting that within this group of patients with an injury of fairly short duration, changes in the articular cartilage or osteochondral junction, or both, had not progressed sufficiently for them to be detected by high definition macroradiography.

In this group of young patients, osteophyte formation on the tibia occurred in the medial compartment only in about half of the injured knees. Although there was some variation, osteophytes tended to appear on the tibia of joints that had had the injury for three years or more. Thus in ACL ruptured knees it would seem that it takes about three to four years from the time of injury for changes in bony features of the medial compartment of the tibial to be detected radiographically. These findings suggest that thickening of the horizontal subchondral trabeculae and the appearance of osteophytes in the tibia are the earliest features, preceding subchondral cortical plate thickening and JSW narrowing. Although appearing at about the same time, no association was found between osteophytes and subchondral bony changes. Whether these features are interrelated remains a matter of speculation in view of the small number of cases. The earlier appearance of bony features, compared with those in cartilage and cortical plate, may be due to its blood supply permitting this tissue to respond to the altered status of the joint. Scintigraphic examination of these joints would help to confirm this suggestion, either from an enhanced isotope localisation detected at the blood pool phase (associated with an increased vascularisation in the subchondral bone) or by the increased isotope uptake at the sites of new bone formation, obtained from the late stage bone scan. Scintigraphy would confirm the presence of actively remodelling bone. ${ }^{36}$ In OA joints, positive scintigraphic scans have identified the presence of growing osteophytes, ${ }^{37}$ remodelling subchondral bone, ${ }^{39}$ and assisted in predicting joints most likely to progress. ${ }^{4041}$

As in knees with early OA, ${ }^{15}{ }^{17}$ none of the above features was detected in the lateral tibial compartment of the ACL ruptured knees. However, the absence of any detectable changes in bone structure in this compartment of the injured knee must cast serious doubt on the part played by the initial insult to bone, detected on MRI. ${ }^{9}{ }^{10}$ Here, the detection of the trauma associated subcortical fractures in the medullary bone, to both the lateral femoral condyle and tibial plateau, ${ }^{8}$ seem not to be associated with any subsequent cancellous bone changes detected radiographically. Indeed, the bone bruising incurred at the time of injury may be a transient event rather than an initiating factor in the onset of OA-like changes in the knee, as suggested elsewhere. ${ }^{11}$

In this preliminary cross sectional study of patients with untreated ACL ruptured knees we found that the earliest anatomical changes, detected radiographically, occur in bone in the subchondral trabeculae and marginal osteophytes. Physical examination confirmed the presence of joint laxity, suggesting an association between the changes in bone and joint instability. Whether these joints would ultimately develop OA, as suggested by others, ${ }^{1-6}{ }^{42}$ is unknown. Nevertheless, this study confirms the need for a larger, prospective longitudinal study of patients with ACL rupture, radiographed sequentially from the time of injury, to test the hypothesis that joint instability alone can induce OA.

The authors wish to express their gratitude to their colleague $\mathrm{Mr}$ Paul Allen FRCS, who provided the patients for this study, to Paul Allen FRCS, who provided the patients for this study, to Mrs Judy Vlahovic for undertaking their radiographic examination, Mrs Barbara Brooksbank for assistance with statistical analysis, and to Ms Sarah Smith for the preparation of the illustrations.

1 McDaniel Jr WJ, Dameron T. The untreated cruciate ligament rupture. Clin Orthop 1983;172:158-63.

2 Matthews DS, Butler DL. The symptomatic anterior cruciate-deficient knee. Part I: The long term functional disability in athletically active individuals. J Bone Joint Surg $\mathrm{Br}$ 1983;65:154-62

3 Sherman M, Warren RF, Marshall J, Savatsky G. A clinical and radiological analysis of 127 anterior cruciate insufficient knees. Clin Orthop 1988;227:229-37.

4 Kannus P, Jarvinen M. Posttraumatic anterior cruciate ligament insufficiency as a cause of osteoarthritis in a knee oint. Clin Rheumatol 1988;8:251-60.

5 Sommerlath K, Lysholm J, Gillquist J. The long-term course after treatment of acute anterior cruciate ligament ruptures. A 9 to 16 years follow up. Am J Sports Med 1991;19:156-62

6 Neyret P, Donell ST, Dejour H. Results of partial menisectomy related to the state of the anterior cruciate ligament. Review at 25 to 35 years. J Bone Joint Surg Br 1993;75:3640.

7 Roos H, Adalberth T, Dahlberg L, Lohmander LS. Osteoarthritis of the knee after injury to the anterior cruciOsteoarthritis of the knee after injury to the anterior cruciate ligament or meniscus: the influence

8 Lohmander LS. Articular cartilage and osteoarthritis-the role of molecular markers to monitor breakdown, repair and disease. J Anat 1994;184:477-92.

9 Rosen M, Jackson DW, Berger PE. Occult osseous lesions documented by magnetic resonance imaging associated with anterior cruciate ligament ruptures. Arthroscopy 1991;7:45-51

10 Speer K, Spritzer CE, Bassett F, Feagin JA, Garrett WE. Osseous injury associated with acute tears of the anterior cruciate ligament. Am J Sports Med 1992;20:382-9.

11 Lohmander LS, Hoerrner LA, Lark MW. Metalloproteinases, tissue inhibitor and proteoglycan fragments in knee synovial fluid in human osteoarthritis. Arthritis Rheum 1993;36:181-9.

12 Buckland-Wright JC. A new high-definition microfocal X-ray unit. Br J Radiol 1989;62:201-8.

13 Buckland-Wright JC, Bradshaw CR. Clinical applications of high-definition microfocal radiography. Br J Radiol 1989; 62:209-17. 
14 Buckland-Wright JC, Macfarlane DG, Lynch JA, Jasani MK, Bradshaw CR. Joint space width measures cartilage thickness in osteoarthritis of the knee: high resolution plain film and double contrast macrora

15 Buckland-Wright JC, Macfarlane DG, Jasani MK, Lynch JA. Quantitative microfocal radiographic assessment of osteoarthritis of the knee from weight bearing tunnel and semi-flexed standing views. J Rheumatol 1994;21:173441.

16 Buckland-Wright JC, Macfarlane DG, Lynch JA, Jasani MK. Quantitative microfocal radiography detects changes in OA knee joint space width in patients in placebocontrolled

17 Buckland-Wright JC, Lynch JA, Macfarlane DG. Fractal signature analysis measures cancellous bone organisation in macroradiographs of patients with knee osteoarthritis. Ann Rheum Dis 1996;55:749-55.

18 Buckland-Wright JC, Macfarlane DG, Lynch JA, Clark B. Quantitative microfocal radiographic assessment of progression in osteoarthritis of the hand. Arthritis Rheum 1990;33:57-65.

19 Buckland-Wright JC. Protocols for precise radio-anatomical positioning of the tibiofemoral and patellofemoral compartments of the knee. Osteoarthritis Cartilage 1995; 3(suppl A):71-80.

20 Williams PL, Bannister LH, Berry MM, Collins P, Dyson M, Dussek JE, et al, eds. Gray's anatomy. 38th ed. New York: Churchill Livingstone, 1995.

21 Lynch JA, Buckland-Wright JC, Macfarlane DG. Precision of joint space width measurement in knee osteoarthritis from digital image analysis of high definition macroradiographs. Osteoarthritis Cartilage 1993:1:209-18.

22 Lynch JA, Hawkes DJ, Buckland-Wright JC. Analysis of texture in macroradiographs of osteoarthritic knees using the fractal signature. Phys Med Biol 1991;36:709-22.

23 Lynch JA, Hawkes DJ, Buckland-Wright JC. A robust and accurate method for calculating the fractal signature of texture in macroradiographs of osteoarthritic knees. Medical ture in macroradiographs of

24 Lundahl T, Ohley WS, Kay SM, Siffert R. Fractional Brownian-motion: a maximum likelihood estimator and its application to imaging texture. IEEE Transactions on Medical Imaging 1986;5:152-61.

25 Ruttimann UE, Webber RL, Hazelrig JB. Fractal dimension from radiographs of periodontal alveolar bone. A possible diagnostic indicator of osteoporosis. Oral Surg Oral Med Oral Pathol 1992;74:98-110.

26 Pentland AP. Fractal based descriptions of natural scenes. IEEE Transactions on Pattern Analysis and Machine Intelligence 1984;6:661-74.

27 Feder J. Fractals. New York: Plenum Press, 1988

28 Majumdar S, Weistein RS, Prased RR, Genant HK. The fractal dimension of trabecular bone: a measure of trabecular structure. Calcif Tissue Int 1993;52:168.
29 Bergot C, Laval-Jeantet AM, Preteux F, Meunier A. Measurement of anisotropic vertebral trabecular bone loss Measurement of anisotropic vertebral trabecular bone loss
during aging by quantitative image analysis. Calcif Tissue during aging by quant

30 Cummings SR, Black D. Should perimenopausal women be screened for osteoporosis? Ann Intern Med 1986;104:81723.

31 Lynch JA, Macfarlane DG, Buckland-Wright JC. Fractal signature analysis measures trabecular organisation in macroradiographs of OA knee patients. Transactions of the Orthopaedic Research Society 1995;20:304.

32 Mow VC, Setton LA, Ratcliffe A, Howell DS, Buckwalter JA. Structure-function relationships of articular cartilage and the effects of joint instability and trauma on cartilage function. In:Brandt KD, ed. Cartilage changes in osteoarthritis. Indianapolis: Indiana University School of Medicine, 1990:22-42.

33 Lohmander LS. Molecular markers of cartilage turnover. A role in monitoring and diagnosis of osteoarthritis. In: Kuettner KE, Goldberg V, eds. Articular cartilage and osteoarthritis. New York: Raven Press, 1992:653-67.

34 Layton MW, Goldstein SA, Goulet RW, Feldkamp LA, Kubinski DJ, Bole GG. Examination of subchondral bone architecture in experimental osteoarthritis by microscopic computed axial tomography. Arthritis Rheum 1988;31: 1400-5

35 Buckland-Wright JC, Macfarlane DG, Lynch J. Relationship between joint space width and subchondral sclerosis in the osteoarthritic hand. A quantitative microfocal radiographic study. J Rheumatol 1992;19:791-8.

36 Christensen SB. Osteoarthrosis, changes in bone, cartilage and synovial membrane in relation to bone scintigraphy. Acta Orthop Scand 1985;56(suppl 214):1-43.

37 Buckland-Wright JC, Macfarlane DG, Fogelman I, Emery P, Lynch JA. Technetium $99 \mathrm{~m}$ methylene diphosphonate bone scanning in osteoarthritic hands. Eur J Nucl Med 1991;18:12-16.

38 Macfarlane DG, Buckland-Wright JC, Emery P, Fogelman I, Clark B, Lynch JA. Comparison of clinical, radionuclide 1, Clark B, Lynch JA. Comparison of clinical, radionuclide and radiographic features of oster

39 McCrae F, Shouls J, Dieppe P, Watt I. Scintigraphic assessment of osteoarthritis of the knee. Ann Rheum Dis 1992;51:938-42.

40 Hutton CW, Higgs ER, Jackson PC, Watt I, Dieppe P. $99 \mathrm{mTC}$ HMDP bone scanning in generalised nodal osteoarthritis. II. The four hour bone scan image predicts radiographic change. Ann Rheum Dis 1986;45:622-6.

41 Dieppe P, Cushnaghan J, Young P, Kirwan J. Prediction of the progression of joint space narrowing in osteoarthritis of
the knee by bone scintigraphy. Ann Rheum Dis 1993;52: 557-63.

42 Hirshman HP, Daniel DM, Miyasaka K. The fate of the unoperated knee ligament injuries. In: Daniel DM, Akeson WH, O'Connor JJ, eds. Knee ligaments: structure, function, injury and repair. New York: Raven Press, 1990:481-503. 